\title{
NEW MEASUREMENTS OF THOUSAND-SEED WEIGHTS OF SPECIES IN THE PANNONIAN FLORA
}

\author{
Töröк, P. ${ }^{*}$, Tóth, E. ${ }^{2}$, Tóth, K. ${ }^{2}$, VAlkó, O. ${ }^{1}$, DeÁK, B. ${ }^{1}$, Kelbert, B. ${ }^{2}$, BÁlint, P. ${ }^{2}$, Radócz, \\ Sz. ${ }^{2}$, Kelemen, A. ${ }^{1}$, Sonkoly, J. ${ }^{2}$, Miglécz, T. ${ }^{2}$, Matus, G. ${ }^{3}$, Takács, A. ${ }^{3}$, Molnár, V. A. ${ }^{3}$, \\ Süveges, K. ${ }^{3}$, Papp, L. ${ }^{4}$, Papp, L. JR. ${ }^{5}$, Tóth, Z. ${ }^{5}$, Baktay, B. ${ }^{6}$, Málnási Csizmadia, G. ${ }^{6}$, \\ Olá́, I. ${ }^{6}$, Peti, E. ${ }^{6}$, Schellenberger, J. ${ }^{6}$, Szalkovszki, O ${ }^{6}$, KIss, ${ }^{2}$ and Tóthmérész, B. ${ }^{1,2}$ \\ ${ }^{1} M T A-D E$ Biodiversity and Ecosystem Services Research Group \\ H-4032 Debrecen, Egyetem tér 1, Hungary; E-mail: *molinia@gmail.com \\ ${ }^{2}$ Department of Ecology, University of Debrecen, H-4002 Debrecen, P.O. Box 400, Hungary \\ ${ }^{3}$ Department of Botany, University of Debrecen, H-4010 Debrecen, P.O. Box 14, Hungary \\ ${ }^{4}$ Botanical Garden, University of Debrecen, H-4010 Debrecen, P.O. Box 40, Hungary \\ ${ }^{5}$ Department of Plant Taxonomy and Ecology, Eötvös Loránd University \\ H-1117 Budapest, Pázmány Péter sétány 1/C, Hungary \\ ${ }^{6}$ Centre for Plant Diversity (CPD), H-2766 Tápiószele, Külsőmező 15, Hungary
}

(Received 6 July, 2015; Accepted 26 August, 2015)

\begin{abstract}
For understanding local and regional seed dispersal and plant establishment processes and for considering the ecotypes and other forms of specific variability, hard data of locally or regionally measured traits are necessary. We provided newly measured seed weight data of 193 taxa, out of which 24 taxa had not been represented in the SID, LEDA or Biolflor databases. Our new measurements and formerly published data of locally collected seed weight records together covers over $70 \%$ of the Pannonian flora. However, there is still a considerable lack in seed weight data of taxonomically problematic genera, even though they are represented in the Pannonian flora with a relatively high number of species and/ or subspecies (e.g. Sorbus, Rosa, Rubus, Crataegus and Hieracium). Our regional database contains very sporadic data on aquatic plants (including also numerous invasive species reported from Hungary and neighbouring countries) and some rare weeds distributed in the southwestern part of the country. These facts indicate the necessity of further seed collection and measurements.
\end{abstract}

Key words: dry storage, hard trait, herbarium, plant trait, restoration, seed database, seed mass

\section{INTRODUCTION}

One of the most easily measurable physical trait of a plant is the weight of its seeds. Seed weight (or referred to also as seed mass) affects the regeneration strategy and the dispersal of plant species both in space (spatial dispersal) and time (development of a seed bank). Seed weight is also strongly related 
to seed predation events (larger seeds are more likely predated), germination processes, seedling establishment and survival (Eriksson 2000). Thus, in the last few decades seed traits (incl. seed weight) became frequently used for explaining crucial dynamical processes in plant communities (Leishman et al. 2001, Moles et al. 2007) and for analysing life trait scenarios (Beaulieu et al. 2007, Moles and Westoby 2003). There is also an increasing trend to collect hard and soft traits into searchable and electronically available databases. This also holds for seed traits, which can be found for the European flora in comprehensive databases, such as BiolFlor (Klotz et al. 2002), BIOPOP (Kleyer 1995), LEDA (Kleyer et al. 2008), TRY (Kattge et al. 2011a, b) the Seed information database SID 7.1 (Kew Botanical Garden, Liu et al. 2008), the Dispersal and diaspore database (Hintze et al. 2013), and the Digital seed atlas of the Netherlands (Cappers et al. 2012). These databases contain data for most of the common European species, especially species with a northwestern or Central European distribution. Species distributed mostly in southern or eastern Europe are generally underrepresented in these databases; thus, providing new, locally collected data on seed weights is a vital task in these regions (see also Csontos et al. 2003, 2007, Török et al. 2013). Furthermore, for understanding local and regional seed dispersal and plant establishment processes and for considering the ecotypes and local specific variability hard data of locally or regionally measured traits are necessary (Cordlandwehr et al. 2013). Beyond the measurement of traits, biological collections, such as seed herbaria and seed banks can store a large number of viable seeds; so they can be used as genetic material for ex situ conservation (Li and Pritchard 2009). Especially hard-coated seeds can retain their viability in dry storage for prolonged periods, even over a century (Molnár et al. 2015).

In the current paper, in addition to Török et al. (2013), we provided new locally measured thousand-seed weight records for 193 species of the Pannonian flora.

\section{MATERIALS AND METHOD}

Seeds and fruits were collected typically between 2010 and 2014 in Hungary (in total 161 items, $82.6 \%$ ). Some other seed sorts were collected in the neighbouring countries, in Romania $(5.7 \%$, RO), Croatia $(0.5 \%, \mathrm{CRO})$, Slovakia $(0.5 \%, \mathrm{SK})$ and Slovenia $(0.5 \%$, SL). In addition, some other sorts originated from outside of the Pannonian Basin: Finland (8.7\%, FIN), Greece $(0.5 \%$, GR), or Turkey $(1.0 \%, T R)$. A fraction $(4.1 \%)$ of the seeds was collected from living plant collections mostly in the Botanical Garden of the University of Debrecen ( $\mathrm{C}$ - see Appendix 1). Seven seed sorts originated from cultivated stands without knowing the origin of seeds used for cultivation. Collection 
dates and locations are reported in Appendix 1. The collected plant material was air-dried then cleaned by hand (also using seed blower and sieves) and dry-stored till measurement. The seeds from fleshy fruits were washed out, then dried. Seed sorts of 100 seeds (germinules or dispersules) were counted to estimate thousand-seed weights. In case of easy-to-harvest and/or common species at least three sorts of 100 seeds were measured. Physically damaged or unfertile seeds were omitted from counting. The air-dry weight of species was measured with $0.0001 \mathrm{~g}$ accuracy using a Sartorius 1702, a Kern ACJ 120-4M or a Kern 410 type analytical balance, up to 200 g. Kern 572 type balance was used above $200 \mathrm{~g}$, with an accuracy of $0.01 \mathrm{~g}$. Dust-seeded species (orchids, Monotropa, Pyrola and Orobanche species) were measured using an AA-200DS (Denver Instrument) type analytical balance with an accuracy of $0.00001 \mathrm{~g}$. Following the seed weight measurements, most of the seed samples were placed at the seed herbarium (containing more than 2,500 records) located at the Department of Ecology (University of Debrecen, DE), at the Soó Rezső Herbarium at the Department of Botany (University of Debrecen, DE), or at the CPD active storage facility (Tápiószele).

\section{RESULTS}

In total, we provided newly measured seed weight data of 193 taxa (Appendix 1), out of which 24 taxa had not been represented in the SID, LEDA or BiolFlor databases.

\section{DISCUSSION}

We are convinced that a database of locally collected seed records and other easily measurable traits would enhance not only the theoretical research of local plant community assembly and functioning, but can help in the practical restoration works. For habitat restoration it is important to use locally harvested plant material (incl. also locally produced or collected seeds; Mijnsbrugge et al. 2010). To compose and fine-tune a proper seed mixture for restoration works and other purposes (i.e. setting the ratios of constituent species) it is necessary to have locally measured seed weights (adjusting or assess the seed numbers sown for respective species). It can also help in the technical implication of sowing, seeds with different sizes, shapes and weights can be hardly sown together with sowing machinery and it requires specific knowledge.

Acknowledgements - The first author was supported by OTKA PD 100192 during the preparation of the manuscript. ET, SR, SJ and AT were supported by the NTP-EFÖ-P-15 pro- 
ject by the Human Capacities Grant Management Office and the Hungarian Ministry of Human Capacities. The study was supported by the SROP-4.2.2.B-15/1/KONV20150001 project. AMV was supported by TÁMOP-4.2.4.A/2-11/1-2012-and OTKA K108992 grants. OV was supported by OTKA PD 111807 grant. Seed collecting and processing activity of CPD were carried out in the frame of "Establishment of the Pannon Seed Bank for the longterm ex situ conservation of Hungarian vascular wild plants" EU LIFE+ project, which was financed by the EU LIFE+ Biodiversity fund and the Hungarian Ministry of Agriculture.

\section{REFERENCES}

Beaulieu, M. M., Moles, A. T., Leitch, I. J., Bennett, M. D., Dickie, J. B. and Knight, C. A. (2007): Correlated evolution of genome size and seed mass. - New Phytol. 173: 422437. http://dx.doi.org/10.1111/j.1469-8137.2006.01919.x

Cappers, R. T. J., Bekker, R. M. and Jans, J. E. A. (2012): Digital seed atlas of the Netherlands. Barkhuis Publishing, Eelde, The Netherlands, 502 pp. http://www.seedatlas.nl

Cordlandwehr, V., Meredith, R. L., Ozinga, W. A., Bekker, R. M., Groenendael, J. M. and Bakker, J. P. (2013): Do plant traits retrieved from a database accurately predict onsite measurements? - J. Ecol. 101: 662-670. http://dx.doi.org/10.1111/1365-2745.12091

Csontos, P., Tamás, J. and Balogh, L. (2003): Thousand seed weight records of species from the flora of Hungary, I. Monocotyledonopsida. - Studia bot. hung. 34: 121-126.

Csontos, P., Tamás, J. and Balogh, L. (2007): Thousand seed weight records of species from the flora of Hungary, II. Dicotyledonopsida. - Studia bot. hung. 38: 179-189.

Eriksson, O. (2000): Seed dispersal and colonization ability of plants - assessment and implications for conservation. - Folia Geobot. 35: 115-123. http://dx.doi.org/10.1007/ bf02803091

Hintze, C., Heydel, F., Hoppe, C., Cunze, S., König, A. and Tackenberg, O. (2013): D3: the dispersal and diaspore database - baseline data and statistics on seed dispersal. - Perspect. Plant Ecol. Evol. Syst. 15: 180-192. http://dx.doi.org/10.1016/j.ppees.2013.02.001

Kattge, J., Ogle, K., Bönisch, G., Díaz, S., Lavorel, S., Madin, J., Nadrowski, K., Nöllert, S., Sartor, K. and Wirth, C. (2011a): A generic structure for plant trait databases. - Methods Ecol. Evol. 2: 202-213. http://dx.doi.org/10.1111/j.2041-210x.2010.00067.x

Kattge, J., Díaz, S., Lavorel, S., Prentice, I. C., Leadley, P., Bönisch, G., Garnier, E., Westoby, M., Reich, P. B., Wright, I. J., Cornelissen, J. H. C., Violle, C., Harrison, S. P., van Bodegom, P. M., Reichstein, M., Enquist, B. J., Soudzilovskaia, N. A., Ackerly, D. D., Anand, M., Atkin, O., Bahn, M., Baker, T. R., Baldocchi, D., Bekker, R. M., Blanco, C., Blonder, B., Bond, W. J., Bradstock, R., Bunker, D. E., Casanoves, F., Cavender-Bares, J., Chambers, J. Q., Chapin III, F. S., Chave, J., Coomes, D., Cornwell, W. K., Craine, J. M., Dobrin, B. H., Duarte, L., Durka, W., Elser, J., Esser, G., Estiarte, M., Fagan, W. F., Fang, J., Fernández-Méndez, F., Fidelis, A., Finegan, B., Flores, O., Ford, H., Frank, D., Freschet, G. T., Fyllas, N. M., Gallagher, R. V., Green, W. A., Gutierrez, A. G., Hickler, T., Higgins, S., Hodgson, J. G., Jalili, A., Jansen, S., Joly, C., Kerkhoff, A. J., Kirkup, D., Kitajima, K., Kleyer, M., Klotz, S., Knops, J. M. H., Kramer, K., Kühn, I., Kurokawa, H., Laughlin, D., Lee, T. D., Leishman, M., Lens, F., Lenz, T., Lewis, S. L., Lloyd, J., Llusià, J., Louault, F., Ma, S., Mahecha, M. D., Manning, P., Massad, T., Medlyn, B., Messier, J., Moles, A. T., Müller, S. C., Nadrowski, K., Naeem, S., Niinemets, Ü., Nöllert, S., Nüske, A., Ogaya, R., Oleksyn, J., Onipchenko, V. G., Onoda, Y., Ordoñez, J., Overbeck, G., Ozinga, W. A., Patiño, S., Paula, S., Pausas, J. G., Peñuelas, 
J., Phillips, O. L., Pillar, V., Poorter, H., Poorter, L., Poschlod, P., Prinzing, A., Proulx, R., Rammig, A., Reinsch, S., Reu, B., Sack, L., Salgado-Negret, B., Sardans, J., Shiodera, S., Shipley, B., Siefert, A., Sosinski, E., Soussana, J.-F., Swaine, E., Swenson, N., Thompson, K., Thornton, P., Waldram, M., Weiher, E., White, M., White, S., Wright, S. J., Yguel, B., Zaehle, S., Zanne, A. E. and Wirth, C. (2011b): TRY - a global database of plant traits. - Glob. Chang. Biol. 17: 2905-2935. http://dx.doi.org/10.1111/j.13652486.2011.02451.x

Kleyer, M. (1995): Biological traits of vascular plants. A database. - Arbeitsber. Inst. f. Landschaftspl. u. Ökol., Univ. Stuttgart, N. F. 2, pp. 1-23.

Kleyer, M., Bekker, R. M., Knevel, I. C., Bakker, J. P., Thompson, K., Sonnenschein, M., Poschlod, P., van Groenendael, J. M., Klimes, L., Klimesová, J., Klotz, S., Rusch, G. M., Hermy, M., Adriaens, D., Boedeltje, G., Bossuyt, B., Dannemann, A., Endels, P., Götzenberger, L., Hodgson, J. G., Jackel, A.-K., Kühn, I., Kunzmann, D., Ozinga, W. A., Römermann, C., Stadler, M., Schlegelmilch, J., Steendam, H. J., Tackenberg, O., Wilmann, B., Cornelissen, J. H. C., Eriksson, O., Garnier, E. and Peco, B. (2008): The LEDA traitbase: a database of life-history traits of Northwest European flora. - J. Ecol. 96: 1266-1274. http://dx.doi.org/10.1111/j.1365-2745.2008.01430.x

Klotz, S., Kühn, I. and Durka, W. (eds) (2002): BiolFlor - eine Datenbank zu biologischökologischen Merkmalen der Gefäßpflanzen in Deutschland. - Schriftenr. f. Vegetationsk. 38: 41-46.

Leishman, M. R. and Murray, B. R. (2001): The relationship between seed size and abundance in plant communities: model predictions and observed patterns. - Oikos 94: 151-161. http://dx.doi.org/10.1034/j.1600-0706.2001.10392.x

Li, D.-Z. and Pritchard, H. W. (2009): The science and economics of ex situ plant conservation. - Trends. Plant. Sci. 14: 614-621. http://dx.doi.org/10.1016/j.tplants.2009.09.005

Liu, K., Eastwood, R. J., Flynn, S., Turner, R. M. and Stuppy, W. H. (2008): Seed information database. (release 7.1, May 2008). - http://www.kew.org/data/sid

Mijnsbrugge, K. V., Bischoff, A. and Smith, B. (2010): A question of origin: where and how to collect seed for ecological restoration. - Basic Appl. Ecol. 11: 300-311. http://dx.doi. org/10.1016/j.baae.2009.09.002

Moles, A. T. and Westoby, M. (2003): Latitude, seed predation and seed mass. - J. Biogeogr. 30: 105-128. http://dx.doi.org/10.1046/j.1365-2699.2003.00781.x

Moles, A., Ackerly, D. D., Tweedle, J. C., Dickie, J. B., Smith, R., Leishman, M. R., Mayfield, M. M., Pitman, A., Wood, J. T. and Westoby, M. (2007): Global patterns in seed size. - Glob. Ecol. Biogeogr. 16: 109-116. http://dx.doi.org/10.1111/j.1466-8238.2006.00259.x

Molnár, V. A., Sonkoly, J., Lovas-Kiss, Á., Fekete, R., Takács, A., Somlyay, L. and Török, P. (2015): Seed of the threatened annual legume, Astragalus contortuplicatus, can survive over 130 years of dry storage. - Preslia 87: 319-328.

Török, P., Miglécz, T., Valkó, O., Tóth, K., Kelemen, A., Albert, Á., Matus, G., Molnár, V. A., Ruprecht, E., Papp, L., Deák, B., Horváth, O., Takács, A., Hüse, B. and Tóthmérész, B. (2013): New thousand-seed weight records of the Pannonian flora and their application in analysing social behaviour types. - Acta Bot. Hung. 55(3-4): 429-472. http:// dx.doi.org/10.1556/ABot.55.2013.3-4.17 
Appendix 1

Thousand-seed weight records of 193 vascular plant taxa; incl. species and some subspecies. Abbreviations: $\mathrm{HS}=$ half-samara, $\mathrm{C}=$ caryopsis, $\mathrm{AC}=$ achaenium, $\mathrm{CC}=$ carcerulus, $\mathrm{SD}=$ seed, $\mathrm{SI}=$ silicula, $\mathrm{D}=$ drupa, $\mathrm{G}=$ glans, $\mathrm{N}=$ nucula, $\mathrm{P}=$ pterodium .

\begin{tabular}{|c|c|c|c|c|c|}
\hline Species & SW & Location & Date & $\mathrm{SN}$ & $\mathrm{M}$ \\
\hline Abies alba & 41.2035 & Mátrafüred & 20.04 .2014 & 3 & SD \\
\hline Acer platanoides & 104.2833 & Farkasgyepü & 10.12.2011 & 4 & HS \\
\hline $\begin{array}{l}\text { Aconitum variegatum } \\
\text { subsp. gracile }\end{array}$ & 0.5846 & Miskolc & 11.08.2011 & 3 & SD \\
\hline Agrostis capillaris & 0.0473 & Bátorliget & 16.07.2013 & 3 & $\mathrm{C}$ \\
\hline Allium moschatum & 1.1213 & Budapest & 10.11.2011 & 3 & SD \\
\hline $\begin{array}{l}\text { Allium rotundum subsp. } \\
\text { waldsteinii }\end{array}$ & 0.8683 & Mezőhegyes & 31.07 .2013 & 4 & SD \\
\hline Allium scorodoprasum & 1.2315 & Kesznyéten & 14.07.2013 & 3 & $\mathrm{SD}$ \\
\hline Andromeda polifolia & 0.1687 & Kirakkajärvi (FIN) & 20.08 .2010 & 3 & $\mathrm{SD}$ \\
\hline Angelica archangelica & 3.1053 & $\begin{array}{l}\text { Sântimbru (Csík- } \\
\text { szentimre) (RO) }\end{array}$ & 03.08 .2013 & 3 & $\mathrm{AC}$ \\
\hline Apera interrupta & 0.0490 & Almásfüzitő & 05.06 .2014 & $\begin{array}{c}0 \\
(56)\end{array}$ & $C^{*}$ \\
\hline Aphanes arvensis & 0.1495 & Debrecen & 11.07.2013 & 3 & $\mathrm{CC}$ \\
\hline Armoracia macrocarpa & 0.6640 & Kesznyéten & 14.07.2013 & 3 & SI \\
\hline Blackstonia acuminata & 0.0135 & Fülöpháza (Bg) & 20.08 .2013 & 3 & SD \\
\hline Borago officinalis & 12.8670 & Debrecen $(C)$ & 29.07.2013 & 1 & $\mathrm{CC}$ \\
\hline Brassica elongata & 0.6517 & Budapest & 10.11.2011 & 3 & $\mathrm{SD}$ \\
\hline Brassica napus & 3.1030 & Kemiö (FIN) & 15.08.2010 & 3 & $\mathrm{SD}$ \\
\hline Bromus pannonicus & 4.6167 & Várvölgy & 21.06 .2013 & 4 & $C^{*}$ \\
\hline Calepina irregularis & 3.6403 & Szeged & 01.06 .2012 & 4 & SD \\
\hline Calla palustris & 3.2010 & Taalinthedas (FIN) & 10.09 .2010 & 3 & SD \\
\hline Campanula rapunculus & 0.0070 & Tiszaújváros & 14.07.2013 & 3 & SD \\
\hline Cardaminopsis arenosa & 0.0703 & Budapest & 20.05 .2013 & 3 & SD \\
\hline Cardaminopsis petraea & 0.0940 & Gyenesdiás & 05.05 .2013 & 1 & SD \\
\hline Carex acutiformis & 0.7387 & Kunfehértó & 19.05.2013 & 4 & $C^{* *}$ \\
\hline Carex appropinquata & 0.9287 & Bátorliget & 10.06 .2011 & 3 & $C^{* *}$ \\
\hline Carex bohemica & 0.2620 & Tiszaadony & 22.07.2012 & 3 & $C^{* *}$ \\
\hline Carex buxbaumii & 0.8843 & Vámospércs & 09.06 .2012 & 3 & $C^{* *}$ \\
\hline Carex cespitosa & 0.4660 & Ispánk & 15.06 .2013 & 3 & $C^{* *}$ \\
\hline Carex diandra & 0.8510 & $\begin{array}{l}\text { Baile (Bálványos) } \\
\text { (RO) }\end{array}$ & 10.08 .2013 & 3 & $C^{* *}$ \\
\hline
\end{tabular}




\begin{tabular}{|c|c|c|c|c|c|}
\hline Species & SW & Location & Date & $\mathrm{SN}$ & M \\
\hline Carex halleriana & 0.4215 & Budapest & 05.06 .2011 & 2 & $C^{* *}$ \\
\hline Carex humilis & 1.3985 & Fót & 11.04 .2014 & 3 & $C^{* *}$ \\
\hline Carex lasiocarpa & 1.7300 & $\begin{array}{l}\text { Lompolonjänkkä } \\
\text { (FIN) }\end{array}$ & 02.08 .2014 & 3 & $C^{* *}$ \\
\hline Carex lasiocarpa & 0.7207 & Sirok & 24.07.2012 & 3 & $C^{* *}$ \\
\hline Carex limosa & 1.9500 & Kirakkajärvi (FIN) & 20.08 .2010 & 3 & $C^{* *}$ \\
\hline Carex otrubae & 1.2960 & Hódmezővásárhely & 22.09 .2012 & 4 & $C^{* *}$ \\
\hline Carex paniculata & 0.6997 & Isaszeg & 28.05 .2011 & 3 & $C^{* *}$ \\
\hline Carex spicata & 1.9250 & Bátorliget & 15.07.2013 & 3 & $C^{* *}$ \\
\hline Catabrosa aquatica & 0.2735 & $\begin{array}{l}\text { Lacu Roşu (Gyilkos- } \\
\text { tó) (RO) }\end{array}$ & 09.08 .2013 & 3 & $\mathrm{C}$ \\
\hline Catalpa bignonioides & 19.7467 & Debrecen & 05.03 .2015 & 3 & $\mathrm{SD}$ \\
\hline Celtis occidentalis & 100.8655 & Debrecen & 26.10 .2010 & 3 & $\mathrm{D}$ \\
\hline $\begin{array}{l}\text { Centaurea jacea subsp. } \\
\text { angustifolia }\end{array}$ & 1.0465 & Hortobágy & 28.07.2012 & 3 & $\mathrm{AC}$ \\
\hline Centaurea pseudophrygia & 2.0300 & $\begin{array}{l}\text { Valea Rece (Hideg- } \\
\text { ség) (RO) (Bg) }\end{array}$ & 04.09 .2013 & 2 & $\mathrm{AC}$ \\
\hline Ceratophyllum demersum & 3.8460 & Tiszapalkonya & 15.08 .2013 & 1 & $\mathrm{AC}$ \\
\hline Chamaenerion angustifolium & 1.8530 & Kemiö (FIN) & 12.08 .2010 & 3 & $\mathrm{SD}^{*}$ \\
\hline Chamaenerion dodonaei & 0.1005 & Dunaalmás & 21.08 .2013 & 3 & $\mathrm{SD}$ \\
\hline Chenopodium botrys & 0.3920 & $\begin{array}{l}\text { Valea Rece (Hideg- } \\
\text { ség) (RO) (Bg) }\end{array}$ & 20.06.2014 & 1 & $\mathrm{SD}$ \\
\hline Cicuta virosa & 1.0840 & Tiszafüred & 20.08.2013 & 3 & $\mathrm{AC}$ \\
\hline Commelina communis & 9.2129 & Debrecen & 13.09.2011 & 3 & SD \\
\hline Coronopus squamatus & 4.3525 & Karcag & 09.07.2013 & 3 & SI \\
\hline Corylus avellana & 1293.8667 & Álmosd & 24.08 .2013 & 3 & G \\
\hline Cotoneaster integerrimus & 8.9120 & Mályinka & 08.06 .2013 & 1 & $\mathrm{SD}$ \\
\hline Crepis pulchra & 0.2630 & Vámospércs & 23.09.1999 & 2 & $\mathrm{AC}$ \\
\hline Crupina vulgaris & 15.2430 & Budapest & 31.05 .2011 & 3 & $\mathrm{AC}$ \\
\hline Cucurbita foetidissima & 43.8320 & Tápiószele & 20.11.2013 & 3 & $\mathrm{SD}$ \\
\hline Cuscuta lupuliformis & 5.9400 & $\begin{array}{l}\text { Tiszaújváros, } \\
\text { Tiszaszederkény }\end{array}$ & 30.08 .2013 & 3 & $\mathrm{SD}$ \\
\hline Dasypyrum villosum & 7.5625 & Paks & 26.08 .2013 & 2 & $C^{*}$ \\
\hline $\begin{array}{l}\text { Dianthus plumarius subsp. } \\
\text { praecox }\end{array}$ & 0.7125 & Bélapátfalva & 10.07.2012 & 1 & SD \\
\hline Dichostylis micheliana & 0.0215 & Lakitelek & 01.10 .2013 & 3 & $\mathrm{C}$ \\
\hline
\end{tabular}




\begin{tabular}{|c|c|c|c|c|c|}
\hline Species & SW & Location & Date & SN & M \\
\hline Digitalis lanata & 0.4667 & Konyár (Bg) & 16.08 .2012 & 3 & SD \\
\hline Draba lasiocarpa & 0.1295 & Krystallopygi (GR) & 23.05 .2012 & 3 & SD \\
\hline Eleocharis uniglumis & 1.2070 & Kesznyéten & 14.07.2013 & 3 & $\mathrm{C}$ \\
\hline Epilobium collinum & 0.0540 & Csatár & 12.08 .2013 & 4 & SD \\
\hline Epipactis palustris & 0.0032 & Monostorpályi & 10.09 .2013 & 3 & SD \\
\hline Eriochloa villosa & 4.5365 & Szentborbás & 25.08 .2013 & 3 & C \\
\hline Erophila spathulata & 0.0049 & Bőcs & 07.04.2015 & 3 & SD \\
\hline Erucastrum gallicum & 0.0965 & Tata & 25.08 .2013 & 3 & SD \\
\hline Euphorbia epithymoides & 1.0980 & $\begin{array}{l}\text { Zgornji Leskovec } \\
\text { (SL) }\end{array}$ & 01.06 .2013 & 1 & SD \\
\hline Euphorbia glareosa & 0.6787 & Veszprém & 22.07.2012 & 4 & SD \\
\hline Euphorbia helioscopia & 2.4055 & Debrecen & 01.05 .2014 & 3 & SD \\
\hline Euphorbia lucida & 1.3425 & Hajdúböszörmény & 17.07.2013 & 3 & SD \\
\hline Euphorbia villosa & 1.4930 & Miskolc & 26.07 .2013 & 3 & SD \\
\hline Festuca arundinacea & 1.8463 & Tápiószele & 16.06 .2012 & 4 & $C^{*}$ \\
\hline Fritillaria meleagris & 1.7335 & Kisar & 20.05 .2014 & 3 & SD \\
\hline Galeopsis tetrahit & 3.5083 & Kemiö (FIN) & 02.09 .2010 & 3 & $\mathrm{CC}$ \\
\hline Galium rubioides & 0.1627 & Pócsmegyer & 12.11 .2011 & 4 & SD \\
\hline $\begin{array}{l}\text { Genista tinctoria subsp. } \\
\text { elata }\end{array}$ & 2.9860 & Telkibánya & 05.08 .2011 & 3 & SD \\
\hline Helictotrichon adsurgens & 6.5267 & Budapest & 01.08 .2011 & 2 & C \\
\hline Helminthia echioides & 0.6225 & Makó & 02.09 .2013 & 3 & $\mathrm{AC}$ \\
\hline Herniaria glabra & 0.0397 & Kemiö (FIN) & 08.10 .2010 & 3 & SD \\
\hline Hesperis matronalis & 1.7313 & Felsőtárkány & 14.08 .2012 & 3 & SD \\
\hline Hieracium bupleuroides & 0.3883 & Bélapátfalva & 10.07.2012 & 3 & $\mathrm{AC}$ \\
\hline Hieracium sabaudum & 0.3540 & Budapest & 31.08 .2011 & 3 & $\mathrm{AC}$ \\
\hline Hierochloe repens & 0.4687 & Budapest & 30.05 .2012 & 3 & $\mathrm{C}$ \\
\hline Hordelymus europaeus & 7.9490 & Miskolc, Ómassa & 10.09 .2013 & 3 & $C^{*}$ \\
\hline Hordeum murinum & 14.1993 & Budapest & 26.06 .2011 & 4 & $\mathrm{C}^{*}$ \\
\hline Inula germanica & 0.0895 & Tápióság & 10.04 .2014 & 3 & $\mathrm{AC}$ \\
\hline Iris arenaria & 6.1003 & Bagamér (Bg) & 13.06 .2013 & 3 & SD \\
\hline Juncus subnodulosus & 0.0140 & Csatár & 12.08 .2013 & 4 & SD \\
\hline Koeleria glauca & 0.1183 & Fülöpháza & 29.06 .2011 & 4 & $C^{*}$ \\
\hline Lathyrus cicera & 29.1607 & Villány & 11.06 .2013 & 3 & SD \\
\hline Lathyrus sphaericus & 23.7883 & Harkány, Terehegy & 08.05 .2013 & 3 & SD \\
\hline
\end{tabular}




\begin{tabular}{|c|c|c|c|c|c|}
\hline Species & SW & Location & Date & $\mathrm{SN}$ & M \\
\hline Lavandula angustifolia & 0.7605 & Debrecen $(\mathrm{C})$ & 31.07 .2013 & 3 & $\mathrm{CC}$ \\
\hline Leymus arenarius & 10.4650 & Strömma (FIN) & 21.08 .2010 & 3 & $\mathrm{C}$ \\
\hline Ligularia sibirica & 1.2110 & $\begin{array}{l}\text { Sâncrăieni (Csík- } \\
\text { szentkirály)(RO) }\end{array}$ & 31.07 .2013 & 1 & $\mathrm{AC}$ \\
\hline Linum austriacum & 1.2237 & Budapest & 19.06.2011 & 4 & SD \\
\hline Loranthus europaeus & 8.1885 & Debrecen & 10.12.2013 & 2 & SD \\
\hline Lotus angustissimus & 0.4750 & Nagyiván & 17.07.2013 & 3 & SD \\
\hline Mahonia aquifolium & 12.0810 & Kunhegyes & 01.07.2014 & 3 & SD \\
\hline Malva pusilla & 1.3000 & Gödöllő & 30.10 .2012 & 4 & SD \\
\hline Malva sylvestris & 1.7700 & Gödöllő & 22.07 .2012 & 4 & $\mathrm{CC}$ \\
\hline Medicago monspeliaca & 0.5025 & Almásfüzitő & 15.06 .2014 & 3 & SD \\
\hline Menyanthes trifoliata & 2.6660 & Lampojänkka (FIN) & 02.08.2014 & 3 & SD \\
\hline Mespilus germanica & 173.262 & Debrecen & 08.12 .2013 & 10 & SD \\
\hline Micromeria thymifolia & 0.2990 & Bélapátfalva & 29.09.2012 & 3 & $\mathrm{CC}$ \\
\hline Minuartia fastigiata & 0.0600 & Dunaalmás & 21.08 .2013 & 3 & SD \\
\hline Minuartia glaucina & 0.0650 & $\begin{array}{l}\text { Pietroasa (Vasaskő- } \\
\text { falva) (RO) }\end{array}$ & 11.07.2010 & 3 & SD \\
\hline Moneses uniflora & 0.0013 & Kolari (FIN) & 31.07 .2014 & 3 & SD \\
\hline Montia perfoliata & 0.3275 & Debrecen (C) & 05.05 .2013 & 3 & SD \\
\hline Myosotis sicula & 0.1530 & Nagyiván & 29.05.2014 & 3 & $\mathrm{CC}$ \\
\hline Nigella damascena & 3.0445 & Debrecen $(\mathrm{C})$ & 16.09 .2013 & 3 & SD \\
\hline Nuphar lutea & 29.406 & Hortobágy & 17.07.2013 & 3 & SD \\
\hline Ononis pusilla & 2.3020 & Budapest & 06.08.2011 & 4 & SD \\
\hline Ophrys fuciflora & 0.0020 & Učka (CRO) & 08.07.2013 & 3 & SD \\
\hline Orchis pallens & 0.0022 & Hajmáskér & 15.06 .2010 & 3 & SD \\
\hline Orobanche cernua & 0.0026 & Almásfüzitő & 05.06.2014 & 3 & SD \\
\hline Orobanche hederae & 0.0055 & $\begin{array}{l}\text { Dizdaroğlu (Sinop) } \\
\text { (TR) }\end{array}$ & 19.07.2014 & 3 & SD \\
\hline Orobanche ramosa & 0.0059 & Sajószöged & 15.09 .2013 & 3 & SD \\
\hline Oxalis acetosella & 1.4575 & $\begin{array}{l}\text { Nagyvisnyó, } \\
\text { Bánkút }\end{array}$ & 07.05 .2015 & 2 & SD \\
\hline Papaver argemone (!) & 0.0675 & $\begin{array}{l}\text { Javorník (CZ) by } \\
\text { Vrbovce (SK) }\end{array}$ & 31.05 .2010 & 3 & SD \\
\hline Papaver hybridum (!) & 0.0465 & Kengyel & 14.05 .2015 & 3 & SD \\
\hline Parietaria diffusa & 0.0643 & Budapest & 15.09 .2013 & 3 & $\mathrm{CC}$ \\
\hline Pedicularis palustris & 0.7093 & $\begin{array}{l}\text { Sâncrăieni (Csík- } \\
\text { szentkirály) (RO) }\end{array}$ & 01.08.2013 & 3 & SD \\
\hline
\end{tabular}




\begin{tabular}{|c|c|c|c|c|c|}
\hline Species & SW & Location & Date & $\mathrm{SN}$ & $\mathrm{M}$ \\
\hline Phacelia tanacetifolia & 2.5410 & Gönc (C) & 02.08 .2013 & 1 & $\mathrm{CC}$ \\
\hline Physospermum cornubiense & 3.8283 & Dömös & 22.08 .2013 & 3 & $\mathrm{AC}$ \\
\hline Pinguicula cf. vulgaris & 0.0150 & Lapland (FIN) & 01.08 .2014 & 3 & SD \\
\hline Plantago altissima & 1.2543 & Lenti & 13.08 .2013 & 4 & SD \\
\hline Platanus $\times$ hybrida & 5.2560 & Debrecen & 24.04 .2014 & 3 & G \\
\hline Poa palustris & 0.1540 & Kesznyéten & 14.07.2013 & 2 & $\mathrm{C}$ \\
\hline Poa remota & 0.2383 & Mátraszentimre & 22.07.2013 & 3 & $\mathrm{C}$ \\
\hline Potamogeton natans & 6.0920 & $\begin{array}{l}\text { Kirakkajärvi } \\
\text { (Kemiö, FIN) }\end{array}$ & 20.08 .2010 & 3 & $\mathrm{CC}$ \\
\hline Potentilla indica & 0.2613 & Miskolc & 05.06 .2015 & 3 & $\mathrm{~N}$ \\
\hline Potentilla micrantha & 0.6160 & Mátrafüred & 18.04.2014 & 3 & $\mathrm{~N}$ \\
\hline Prenanthes purpurea & 0.4415 & Répáshuta & 12.08 .2012 & 2 & $\mathrm{AC}$ \\
\hline Primula farinosa & 0.0433 & Budapest (C) & 08.07.2012 & 3 & SD \\
\hline Pseudofumaria lutea & 1.1035 & Debrecen $(\mathrm{C})$ & 21.08 .2013 & 3 & $\mathrm{CC}$ \\
\hline $\begin{array}{l}\text { Pseudolysimachion } \\
\text { longifolium }\end{array}$ & 0.0613 & Álmosd & 15.08 .2013 & 3 & SD \\
\hline Pyrola rotundifolia & 0.0013 & Kemiö (FIN) & 02.09 .2010 & 3 & SD \\
\hline Puccinellia distans & 0.1443 & Gödöllő & 02.07 .2012 & 4 & $C^{*}$ \\
\hline Quercus palustris & 1455.2577 & Körmend (C) & 22.12.2012 & 1 & G \\
\hline Ranunculus bulbosus & 3.3640 & Várvölgy & 21.06 .2013 & 4 & SD \\
\hline Ranunculus lingua & 1.1175 & Kesznyéten & 14.07 .2013 & 3 & $\mathrm{~N}$ \\
\hline Ranunculus nemorosus & 0.2480 & $\begin{array}{l}\text { Valea Rece (Hideg- } \\
\text { ség) (RO) (Bg) }\end{array}$ & 28.07.2014 & 1 & $\mathrm{~N}$ \\
\hline Ranunculus psilostachys & 0.2330 & $\begin{array}{l}\text { Nagyharsány (Deb- } \\
\text { recen, Bg) }\end{array}$ & 22.05 .2014 & 3 & $\mathrm{~N}$ \\
\hline Rheum rhabarbarum & 21.7447 & Taalinthedas (FIN) & 10.09 .2010 & 3 & $\mathrm{CC}$ \\
\hline Rorippa sylvestris & 0.0470 & Érd & 30.10 .2011 & 4 & SD \\
\hline Rosa gallica & 26.8135 & Tarcal & 19.10 .2013 & 3 & $\mathrm{~N}$ \\
\hline Rosa villosa & 13.7653 & Budapest & 11.11.2011 & 3 & $\mathrm{~N}$ \\
\hline Salix pentandra & 0.3317 & Bátorliget & 29.10 .2011 & 3 & $\mathrm{SD}$ \\
\hline Salvia $\times$ betonicifolia & 0.9220 & Kondoros & 10.06 .2012 & 2 & $\mathrm{SD}$ \\
\hline Sambucus racemosa & 1.7585 & Miskolc & 06.07 .2013 & 3 & $\mathrm{SD}$ \\
\hline Sanguisorba minor & 6.1557 & Budapest & 31.05 .2011 & 3 & $\mathrm{~N}$ \\
\hline Saxifraga bulbifera & 0.0133 & Bojt & 08.05 .2014 & 3 & SD \\
\hline Scabiosa canescens & 0.7630 & Dunaalmás & 25.08 .2013 & 1 & $\mathrm{AC}$ \\
\hline Scandix pecten-veneris & 19.7110 & Hadim (Konya) (TR) & 08.06.2014 & 3 & $\mathrm{AC}$ \\
\hline
\end{tabular}




\begin{tabular}{|c|c|c|c|c|c|}
\hline Species & SW & Location & Date & SN & $\mathrm{M}$ \\
\hline Schoenoplectus tabernaemontani & 1.9265 & Szabadkígyós & 18.07.2013 & 3 & $\mathrm{C}$ \\
\hline Scorzonera parviflora & 3.4090 & Budapest & 30.05 .2012 & 3 & $\mathrm{AC}$ \\
\hline Sedum sexangulare & 0.0157 & Pomáz & 20.07.2011 & 4 & SD \\
\hline Sedum spurium & 0.0670 & Taalinthedas (FIN) & 07.09.2010 & 3 & SD \\
\hline $\begin{array}{l}\text { Sedum urvillei subsp. } \\
\text { hillebrandtii }\end{array}$ & 0.1915 & Fülöpháza & 23.09 .2013 & 3 & SD \\
\hline Senecio paludosus & 0.5377 & $\begin{array}{l}\text { Sântimbru (Csík- } \\
\text { szentimre) (RO) }\end{array}$ & 01.08 .2013 & 3 & $\mathrm{AC}$ \\
\hline Seseli peucedanoides & 0.9840 & Felsőtárkány & 16.08 .2013 & 3 & $\mathrm{AC}$ \\
\hline Sesleria sadleriana & 2.2257 & Budapest & 23.05.2011 & 3 & $\mathrm{C}$ \\
\hline Sesleria uliginosa & 0.8163 & $\begin{array}{l}\text { Tuşnadu Nou (Új- } \\
\text { tusnád) (RO) }\end{array}$ & 04.08 .2013 & 3 & $\mathrm{C}$ \\
\hline Sicyos angulatus & 55.8015 & $\begin{array}{l}\text { Tiszaújváros, } \\
\text { Tiszaszederkény }\end{array}$ & 30.08 .2013 & 3 & SD \\
\hline Silene bupleuroides & 0.5333 & Budapest & 10.11.2011 & 3 & SD \\
\hline Silene borysthenica & 0.2257 & Bócsa & 01.11 .2010 & 3 & SD \\
\hline Silene dichotoma & 0.7810 & Bőcs & 14.07.2013 & 3 & SD \\
\hline Silene viridiflora & 0.3080 & Bátorliget & 15.07.2013 & 3 & SD \\
\hline Sisymbrium loeselii & 0.5945 & Kányaháza & 25.07.2011 & 3 & SD \\
\hline Spergula arvensis & 0.4553 & Strömma (FIN) & 21.08 .2010 & 3 & SD \\
\hline Stellaria palustris & 0.4370 & Kesznyéten & 14.07.2013 & 1 & SD \\
\hline Succisella inflexa & 1.4563 & Lakócsa & 25.08 .2013 & 3 & $\mathrm{AC}$ \\
\hline Tamus communis & 14.8610 & Keszthely & 15.09 .2014 & 2 & SD \\
\hline Taraxacum serotinum & 0.8580 & Isaszeg & 26.08 .2011 & 2 & $\mathrm{AC}$ \\
\hline Tephroseris crispa & 0.4300 & Mátraszentimre & 22.07 .2013 & 3 & $\mathrm{AC}$ \\
\hline Teucrium scordium & 0.3247 & Szatymaz & 27.08.2012 & 4 & SD \\
\hline Thalictrum flavum & 1.3267 & Zákányszék & 17.08.2011 & 4 & $\mathrm{CC}$ \\
\hline Thlaspi alliaceum & 0.5420 & Dobronhegy & 15.05 .2014 & 1 & SD \\
\hline Thymus praecox & 0.6390 & Budapest & 28.06 .2012 & 3 & $\mathrm{CC}$ \\
\hline Tragopogon floccosus & 4.6860 & Budapest & 30.09 .2013 & 2 & $\mathrm{AC}$ \\
\hline Trifolium medium & 1.8420 & Gönc & 30.07 .2013 & 3 & SD \\
\hline Triglochin maritimum & 0.2557 & Kunpeszér & 25.09 .2012 & 4 & SD \\
\hline Triglochin maritimum & 0.4830 & Kemiö (FIN) & 20.08.2010 & 3 & SD \\
\hline Trigonella caerulea & 1.8345 & Almásfüzitő & 15.06 .2013 & 3 & SD \\
\hline Turgenia latifolia & 36.1210 & Körösladány & 18.07.2013 & 3 & $\mathrm{AC}$ \\
\hline Ulmus pumila & 11.0445 & Debrecen & 25.04 .2014 & 3 & $\mathrm{P}$ \\
\hline
\end{tabular}




\begin{tabular}{lclccc} 
Species & SW & Location & Date & SN & M \\
\hline Ulmus pumila & 5.4505 & Debrecen, Szepes & 28.04 .2014 & 3 & P \\
Urtica urens & 0.4540 & Debrecen & 18.06 .2012 & 3 & CC \\
Veratrum nigrum & 4.0107 & Keszthely & 07.09 .2012 & 3 & SD \\
Verbascum densiflorum & 0.0837 & Hódmezővásárhely & 13.09 .2012 & 4 & SD \\
Veronica praecox & 0.1170 & Budapest & 20.04 .2014 & 3 & SD \\
Viburnum lantana & 37.9590 & Ganna & 17.07 .2011 & 3 & SD \\
Viola ambigua & 2.4100 & Mezőhegyes & 06.08 .2011 & 4 & SD \\
Viola canina subsp. montana & 0.2360 & Mátrafüred & 19.05 .2013 & 1 & SD \\
Viola reichenbachiana & 1.1780 & Telkibánya & 30.07 .2013 & 2 & SD \\
Viola suavis & 2.4223 & Tápiószele & 12.05 .2011 & 4 & SD \\
\hline
\end{tabular}

$\mathrm{SW}=$ thousand-seed weights $(\mathrm{g})$, Date $=$ day.month.year, $\mathrm{SN}=$ number of hundred-seed sorts measured; without respective sorts of hundred seeds, the number of seeds measured is reported in brackets.

Location: for collections outside of the border of Hungary, country codes are given in brackets. $\mathrm{M}=$ measured morphological unit: ${ }^{*}=$ measured with appendix, ${ }^{* *}=$ measured in utriculus. Altogether 24 records missing from SID (Kew), LEDA and BiolFlor databases are indicated with boldface. The location of the botanical garden or the place of cultivation and " $(\mathrm{C})$ " are inserted when the seeds originated from a botanical garden or cultivation with unknown source of seed was used for cultivation. With known origin of the seed used for cultivation, the location and "(Bg)" are inserted. (!) - Green capsules collected, maturated later - thus, much smaller seeds can be expected than in the literature 